\title{
COMPARISON AND ANALYSIS OF APPLICATION EFFECT OF TRADITIONAL PAPER OPERATION METHOD AND DIGITAL INFORMATION SYSTEM IN HEMODIALYSIS
}

\author{
Xiaowang Chen, Yucheng Su*, Mingwei Huang \\ Affiliated Hospital of Guilin Medical University, China. \\ *Corresponding Author email: 24547350@qq.com
}

This is an open access article distributed under the Creative Commons Attribution License, which permits unrestricted use, distribution, and reproduction in any medium, provided the original work is properly cited

\section{ARTICLE DETAILS}

\section{Article History:}

Received 12 November 2017 Accepted 12 December 2017 Available online 1 January 2018

\begin{abstract}
Objective To investigate the effect of traditional paper operation methods and digital information systems on improving clinical work efficiency. Methodology A number of clinical controlled studies and questionnaire surveys were conducted to evaluate the operation of the hemodialysis information electronic management system, its impact on dialysis work efficiency and dialysis quality, and the satisfaction of doctors and patients, and achieved hemodialysis centers. Electronic information management can save costs and control operating expenses while liberating people and improving work efficiency. Results The information electronic management system helps improve the safety of hemodialysis treatment. With the use of electronic information management, the integrity of the records of hemodialysis medical records has been significantly improved, and the authenticity has been better guaranteed. The implementation rate of doctor's orders reaches $100 \%$, and medical errors are reduced, making it easier for traceability and quality control. Conclusion The use of digital information system in hemodialysis can improve the work quality and work efficiency of the hemodialysis room and improve the satisfaction of doctors and patients as a whole. Therefore, digital information systems have positive application value in hemodialysis..
\end{abstract}

\section{KEYWORDS}

Digital information system, paper operation method, hemodialysis, satisfaction, work efficiency.

\section{INTRODUCTION}

At present, most of the countries at home and abroad have used the field inspection and dialysis registration system of the health supervision department to evaluate the operation and medical conditions of each hemodialysis center. Field inspections are limited in their effectiveness due to limited inspection time and geographical location. Health regulatory authorities must achieve long-term, systematic management, rely more on the establishment of a dialysis registration system, and expect to effectively assess the treatment level and management quality of each dialysis center through the data of the system [1]. This kind of supervision mode can realize effective assessment. The premise is that each Hemodialysis Center needs to input medical information into the registration management system in a timely, effective and comprehensive manner, and it can be truly credible. Therefore, this management model needs to rely on the strict self-discipline and responsibility of each dialysis center.

At the same time, this management model also consumes a lot of manpower to manually input medical-related data, which is unfavorable for improving management efficiency and saving medical costs. The burden of work is heavy and tedious, the quality of medical care cannot be effectively monitored, and in the face of ever-increasing patient groups and increasingly stringent quality control requirements, how to make the improvement of management efficiency keep pace with the scale expansion is a serious challenge for clinical work [2]. In order to resolve these conflicts and respond to job challenges, the electronic management system for hemodialysis information has gradually gained attention as an electronic management system. Looking at the field of medical science, it has always been at the forefront of science and technology. The development of information technology has led to the emergence of digital hospitals.
The digital hospital information system (HIS), the electronic data management system of the clinical laboratory, the digital data management system of the imaging department, and the various clinical departments of the operating room and urology department applied to the information management system enabled us to see the electronic information management system of the hemodialysis center [3]. Good application prospects. Digital hospital management refers to the use of computers and network technologies to organically integrate all hospital business information and management information, realize the collection, transmission, storage, utilization, and sharing of this information to the maximum extent, and realize the hospital information system with optimized medical procedures. Its features are paperless, filmless, and wireless networking.

The domestic status quo is that the digitalization of the operating room and the medical and laboratory departments such as imaging and inspection is relatively at the forefront. This is also due to the characteristics of its own batch and relatively uniform conclusions. These successful experiences give us a theoretical basis and confidence in applying this discipline [4]. In recent years, many domestic hospitals have tried to establish a variety of hemodialysis electronic management database, but still need to manually enter information, time-consuming and laborious, accuracy cannot be guaranteed. Therefore, the hemodialysis center urgently needs to manage information electronic management systems that are more effective and easier to operate.

\section{MATERIALS AND METHODS}

\subsection{Normal information}

Select 50 patients who can continue hemodialysis in our hospital for more than 2 years, and randomly divide them into 2 groups, namely traditional paper operation group and digital information system group [5]. Each 
group is randomly assigned 5 nurses, including 10 nurses. He has worked in hemodialysis for more than five years, mastered the relevant operations of the entire dialysis process, and has used the electronic information management system more than six months.

All traditional paper operations groups use medical handwritten dialysis log sheets, and each patient enters dialysis prescriptions on the fly. The digital information system group automatically calculates the ultrafiltration volume and downloads the dialysis prescription. The dialysis record sheet is automatically generated after each treatment.

\subsection{Method}

Through controlled clinical studies, maintenance hemodialysis patients in hemodialysis centers were divided into two groups. The control group was managed by traditional paper operations, and the observation group was administered electronic information management [6]. By monitoring the patient's blood pressure, heart rate, and the time spent on arterial and venous pressures, the impact of the electronic information management system for hemodialysis on work efficiency was analyzed.

At the same time, questionnaires were used to survey the satisfaction and adaptability of the hemodialysis information electronic management system of all dialysis patients and medical staff of the Center to assess the acceptance and improvement of the information management system of hemodialysis information.

The content of the investigation includes the patient questionnaire including the demographic information of the patient, the investigation of the degree of adaptation of the system, and the satisfaction survey of hemodialysis. These include: Overall evaluation of the use of digital information systems (very satisfied, satisfied, basically satisfied, not satisfied), the degree of adaptation (very adapted, adapted, basic adapted, not adapted). The medical staff questionnaire includes the satisfaction and awareness of the hemodialysis information management system, the comparison with the paper operation methods (including the patient management process and report output), and the frequency of the use of electronic system reports in actual work.

The survey method used a questionnaire survey method. The members of the research team designed the "Hematology Room Patient Satisfaction Survey Form" and the "Hospital Workers Satisfaction Survey Form" by consulting the literature and consulting experts and revised and improved through pre-investigation. Investigators passed 168 copies, distributed 74 questionnaires for medical staff, and collected 74 valid questionnaires.

\subsection{Observation indicators}

Analysis of the impact of electronic information management on the efficiency of hemodialysis work, including differences in the time of dialysis management and differences in the timing of vital sign detection. Compared with the conventional management team, the digital information system group has significantly reduced the time taken for dialysis prescriptions to be issued and confirmed, the time for the detection of vital signs to be completely saved, and significantly improved work efficiency and labor costs.

\subsection{Satisfaction survey}

Satisfaction survey results show that most patients are satisfied or basically satisfied with the use of electronic information management systems and can adapt to the operation and management of electronic systems. The questionnaires of medical staff showed that most of the medical staff can master the workflow of the digital system and think that using the system in the clinical work can complete the daily work and basic maintenance needs. Compared with the paper operation method, the patient management process is greatly simplified, the report output speed is significantly improved, and the frequency of the use of digital system reports in the actual work is gradually increasing.

\subsection{Statistical analysis}

SPSS 20.0 statistical software was used to process the data, and the measurement data was represented by $\overline{\mathrm{X}} \pm \mathrm{S}$; the analysis of variance was used to compare the two groups. The count data was represented by the number of cases (n) and percentage (\%). The difference was statistically significant at $\mathrm{P}<0.05$.

\section{RESULT}

Comparison of relevant indicators between observation group and control group:

Table 1: Comparison of relevant indicators between observation group and control group:

\begin{tabular}{|c|c|c|c|}
\hline \multicolumn{2}{|l|}{ Category } & Control group & $\begin{array}{l}\text { Observation } \\
\text { group }\end{array}$ \\
\hline \multicolumn{2}{|c|}{ Record integrity and authenticity } & $<100 \%$ & $100 \%$ \\
\hline \multicolumn{2}{|c|}{ Doctor's execution } & $<100 \%$ & $100 \%$ \\
\hline \multicolumn{2}{|c|}{ Work efficiency (minutes/persons) } & 5 & 1.5 \\
\hline \multirow{5}{*}{$\begin{array}{l}\text { Satisfaction } \\
\text { survey } \\
\text { (patient) }\end{array}$} & Very satisfied & - & $70 \%$ \\
\hline & Satisfaction & - & $20 \%$ \\
\hline & $\begin{array}{l}\text { Basically } \\
\text { satisfied }\end{array}$ & - & $10 \%$ \\
\hline & Not satisfied & - & $0 \%$ \\
\hline & Very satisfied & - & $80 \%$ \\
\hline \multirow{3}{*}{$\begin{array}{l}\text { Satisfaction } \\
\text { survey } \\
\text { (medical staff) }\end{array}$} & Satisfaction & - & $10 \%$ \\
\hline & $\begin{array}{l}\text { Basically } \\
\text { satisfied }\end{array}$ & - & $0 \%$ \\
\hline & Not satisfied & - & $0 \%$ \\
\hline
\end{tabular}

In this study, before the operation of the information electronic management system, the treatment records of the Center were checked one by one and it was found that the existence date, anticoagulants, blood flow, purification methods, conductance, medical staff signatures, etc. were all missing records. Sexuality cannot be confirmed by $100 \%$. After the information management system is used, these items are automatically recorded by the system, and the record integrity is significantly improved to reach $100 \%$. Once the data is entered into the database, it cannot be artificially modified, and the authenticity is guaranteed.

In the paper treatment records, the strength of the doctor's execution cannot be assessed because the records are complete and accurate and difficult to verify. However, after the information electronic management system is running, each doctor's prescription, including dialysis prescriptions and temporary medical orders, can be sent to the patient's hemodialysis machine terminal in a timely and accurate manner. Therefore, whether or not nurses are executed, when they are performed, and which nurses are performing such information will be fed back to the electronic management monitoring system and database system in a timely manner to ensure that the implementation rate of the doctor's orders reaches $100 \%$, and medical errors are reduced, making it easy for traceability and quality control.

Intelligent information management can effectively improve the efficiency of hemodialysis. Compared with the conventional management group, the dialysis prescription and confirmation time of the electronic management team were significantly reduced, and the blood pressure, heart rate, and arteriovenous pressure can be automatically monitored to effectively save the operation time of the medical staff. After the application of information electronic management system, there was no obvious change in the quality of dialysis. The improvement of blood pressure may be related to more timely monitoring and alarm of blood pressure. The improvement of nutrition score and the increase of urea clearance may be related to the guarantee of hemodialysis time, and the dialysis is more adequate. related.

When the patients in the routine management group were on the machine, the dialysis prescription input time was approximately 5 minutes/person, and the dialysis prescription download confirmation time of the electronic management team was 1.5 minutes/person, in which the dialysis prescription was issued and confirmed, including the ultrafiltration volume calculation time. The setting time of dialysis machine parameters was significantly reduced, and the difference between the two groups was statistically significant. 
In the survey of 74 effective patient satisfaction surveys, the basic situation of the surveyed subjects was that the average age was 58.2 years, with 43 males and 34 females, with an average of 5.2 years of dialysis. The results showed that most of the patients were satisfied or basically satisfied with the use of the hemodialysis information electronic management system. At the same time, the questionnaire surveyed the patient's adaptability. The results showed that $87.3 \%$ of patients could adapt to the operation and management of the information electronic management system.

In a survey of 74 effective health care workers' satisfaction surveys, surveys of 10 health care workers showed that medical staff can master the work flow of the digital system proficiently, and their awareness of the electronic information management system for hemodialysis reached $100 \%$. Satisfied $80 \%$, Satisfied $10 \%$, Basically satisfied $10 \%$, Unsatisfied $0 \% .100 \%$ of medical staff believe that using the system in clinical work can accomplish daily work and basic maintenance needs. Compared with the paper operation method, the patient management process is greatly simplified, the report output speed is significantly improved, and the frequency of the use of digital system reports in the actual work is gradually increasing.

Both the patient satisfaction survey results and the medical staff questionnaire survey results show that all types of people can well adapt to and apply the information electronic management system and have a higher degree of satisfaction with the system.

\section{CONCLUSION}

This study shows that the clinical application of the hemodialysis electronic management system improves the efficiency of dialysis, improves the quality of dialysis, and improves the management level of the hemodialysis center.

\section{ACKNOWLEDGEMENT}

Guangxi young teachers in the basic ability to enhance the project (KY2016LX229).

\section{REFERENCES}

[1] Liyan, G. 2015. Analysis of impact of implementation process management on the quality of hemodialysis room. Journal of Traditional Chinese Medicine Management, 31 (08), 117-119.

[2] Jun, T., Hong, L., Lanping, H. 2016. Research on the effect of total quality management tools in the continuous improvement of quality of care in Hemodac. Chinese Journal of Integrative Medicine on Cardiovascular Disease Electron, 4 (14), 74-75.

[3] Danhua, T. 2007. Hospital information system HIS and its application. China Medical Device Information, 13 (9).

[4] Kai, S., Yanming, X., Wei, Z., Bo, F., Yan, Z. 2018. A Study of TCM Syndrome Features of Patients with Real World Gastric Malignant Tumors Based on Hospital Information System. Chinese Journal of Information on Traditional Chinese Medicine, 03.

[5] Yanlin, H., Qiyan, C. 2017. Main types of hospital information system software maintenance and precautions Medical Equipment, 06.

[6] Xiangjie, L. 2017. Analysis of Hospital Information System Software Maintenance. Computer Programming Skills and Maintenance, 05. 